\title{
Distribución histórica y actual de la lagartija Liolaemus pictus (Dumeril \& Bibron 1837) (Liolaemidae) y nuevo límite continental sur de distribución
}

\section{Historical and current distribution of the lizard Liolaemus pictus (Dumeril \& Bibron 1837) (Liolaemidae) and new continental southern limit of distribution}

\author{
Iván M. Vera-Escalona ${ }^{1 *}$, Tania Coronado ${ }^{1}$, Carla Muñoz-Mendoza ${ }^{1}$ \& Pedro F. Victoriano ${ }^{2}$ \\ ${ }^{1}$ Programa Magíster en Ciencias-mención Zoología. Universidad de Concepción, Casilla 160-C, Concepción, Chile. \\ ${ }^{2}$ Departamento de Zoología. Facultad de Ciencias Naturales y Oceanográficas. Universidad de Concepción, Casilla 160-C, \\ Concepción, Chile. \\ *E-mail: ivvera@udec.cl
}

\begin{abstract}
RESUMEN
Basado en los registros de la literatura y datos obtenidos de nuestras colectas, discutimos la distribución actual de Liolaemus pictus. La comparación de los resultados indica que esta especie no ha sido detectada en la depresión intermedia de Chile desde 1934. Lo anterior implica que dichas poblaciones se han extinguido, seguramente como consecuencia de la deforestación. Al mismo tiempo, extendemos el límite sur de la distribución conocida de L. pictus hasta los $44{ }^{\circ} \mathrm{S}$ en la Región de Aysén.
\end{abstract}

\begin{abstract}
Based on literature records and data gathered during our fieldwork, we discuss the current distribution of Liolaemus pictus. The comparison of results shows that this species has not been detected since 1934 in the southern Chilean intermediate depression, implying populations' extinction associated to deforestation. In addition, we found individuals of $L$. pictus in Aysén Region, extending its southern distribution up $44^{\circ} \mathrm{S}$.
\end{abstract}

Liolaemus es un género de lagartijas sudamericanas de la familia Liolaemidae que presenta un alto número de especies, algunas de ellas presentes en Chile (Etheridge \& Espinoza 2000). Una de estas especies es Liolaemus pictus (Dumeril \& Bibron, 1837), lagartija de tamaño mediano categorizada como vulnerable (Nuñez et al. 1997, Ortiz et al. 1990). Se distribuye en la provincia de Río Negro en Argentina, en Chile continental entre Vilches Alto (35 $\left.35^{\circ} \mathrm{S} 71^{\circ} 05^{\prime} \mathrm{O}\right)$ y la Reserva Nacional Futaleufú $\left(43^{\circ} 15^{\prime} \mathrm{S} 71^{\circ} 47^{\prime} \mathrm{O}\right)$ y en Chile insular en las islas del archipiélago de Chiloé y en Isla Mocha (Veloso \& Navarro 1988, Pincheira-Donoso \& Nuñez 2005, Elgueta et al. 2006). Habita áreas boscosas desde el nivel del mar hasta aproximadamente los 1500 msnm, encontrándose sobre troncos y árboles (DonosoBarros 1966). Su alimentación es insectívora y frugívora, siendo un importante dispersor de semillas de plantas y arbustos. (Willson et al. 1996a, 1996b, Ortiz 1974). Parte de su área de distribución, la depresión intermedia de Chile, ha sido modificada durante los últimos 150 años (Haig et al. 1946), generando una pérdida mayor del hábitat boscoso que podría afectar la presencia de L. pictus.
A través de muestreos realizados entre los años 2004-2009 (Apéndice 1), se han determinado áreas de presencia $\mathrm{y}$ ausencia que se contrastan con los registros consignados en la literatura del siglo pasado (Fig. 1). La información obtenida en terreno durante la presente década muestra la ausencia de L. pictus en la depresión intermedia de Chile, desde aproximadamente los $38^{\circ} \mathrm{S}$. En algunos sectores, como son los alrededores de Temuco y Osorno (Hellmich 1934), esta especie no ha sido detectada desde hace más de 50 años. Este patrón de ausencia de ejemplares ya había sido señalado por Smith-Ramirez et al. (2005) para la vertiente continental de la Cordillera de Nahuelbuta. Esto coincide con la ausencia de bosques en la depresión intermedia (Figura 2) debido a las alteraciones de origen antrópico que han tenido lugar desde 1950 (Heusser 2003). Es así que estos resultados apoyan la sugerencia de Ibarra-Vidal (1989) en cuanto a que las alteraciones producto de la deforestación $\mathrm{y}$ el monocultivo de especies forestales han impactado negativamente a la herpetofauna chilena. 
Los muestreos realizados nos han permitido además ampliar el límite sur de la distribución conocida de esta especie en base a ejemplares de Canal Garrado (435' $\mathrm{S}$ ) en la Región de Aysén (Fig. 1), en un localidad más austral que los registros reportados por Elgueta et al. (2006). Esto confirma la presencia de L. pictus en ambientes de bajos promedios térmicos y alta pluviosidad, lo cual ratifica sus rangos particulares de tolerancia y requerimientos térmicos dentro del género Liolaemus (Labra et al. 2009, Vidal \& Sabat 2010).
Dadas las condiciones actuales de la depresión intermedia, la ausencia de ejemplares de L. pictus en áreas para las cuales existen registros históricos, parece ser un ejemplo de cómo las alteraciones antrópicas presentan una de las mayores amenazas a la herpetofauna (Vitousek et al. 1997), provocando una considerable pérdida de biodiversidad (Bell \& Donnelly 2006). Por ello, notificar la ausencia de ejemplares en áreas donde solían existir es de suma

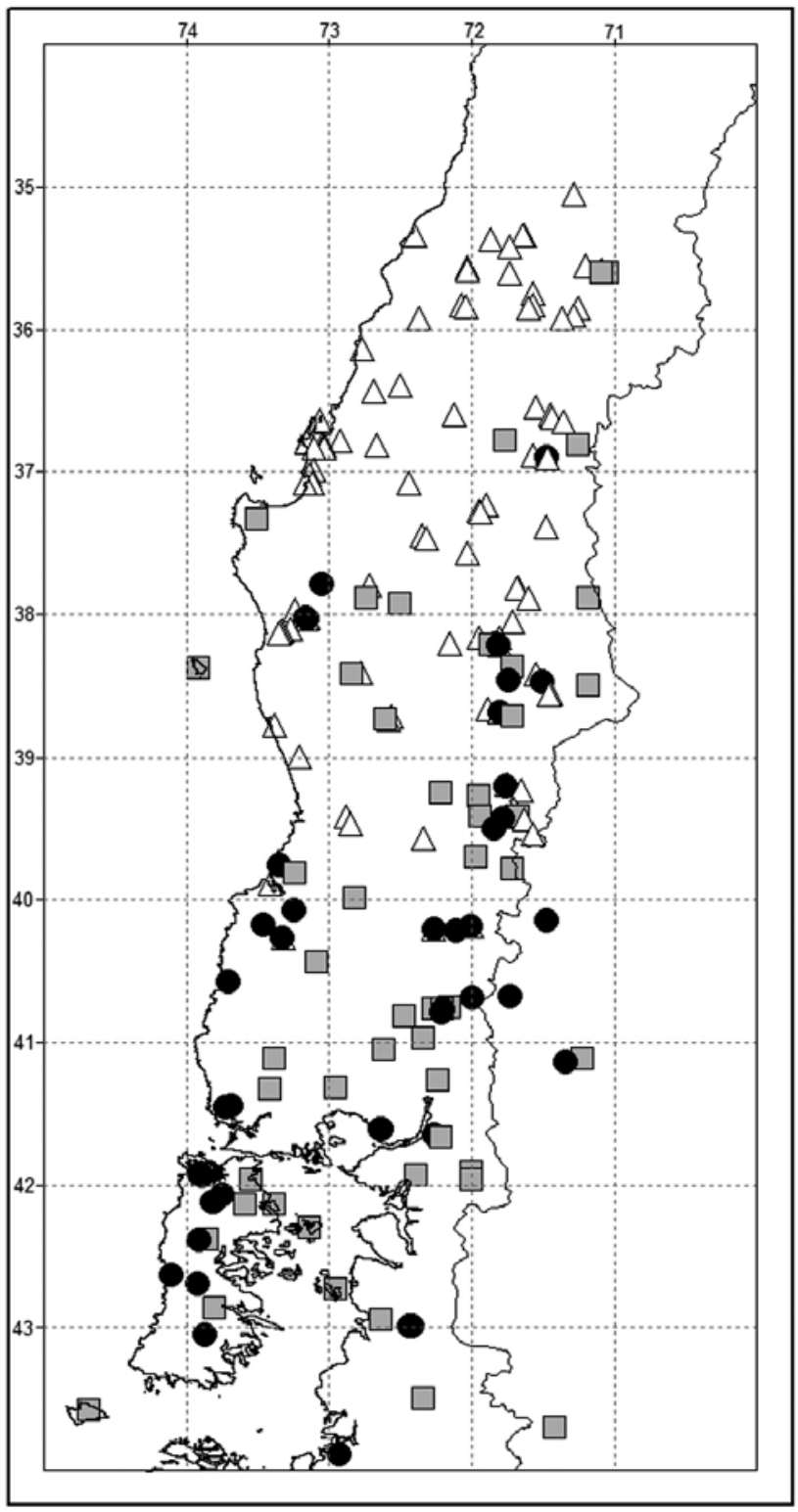

Figura 1. Distribución de Liolaemus pictus. Los círculos negros indican puntos muestreados por los autores donde se encontraron ejemplares. Los triángulos blancos indican puntos muestreados por los autores donde no se detectó la especie. Los cuadros grises indican puntos donde la literatura informa la ocurrencia de L. pictus.

Figure 1. Distribution of Liolaemus pictus. Black circles show localities sampled by the authors where individuals were found. White triangles show localities sampled by the authors where L. pictus was not found. Grey squares show points of the intermediate depression with literature recording localities. 


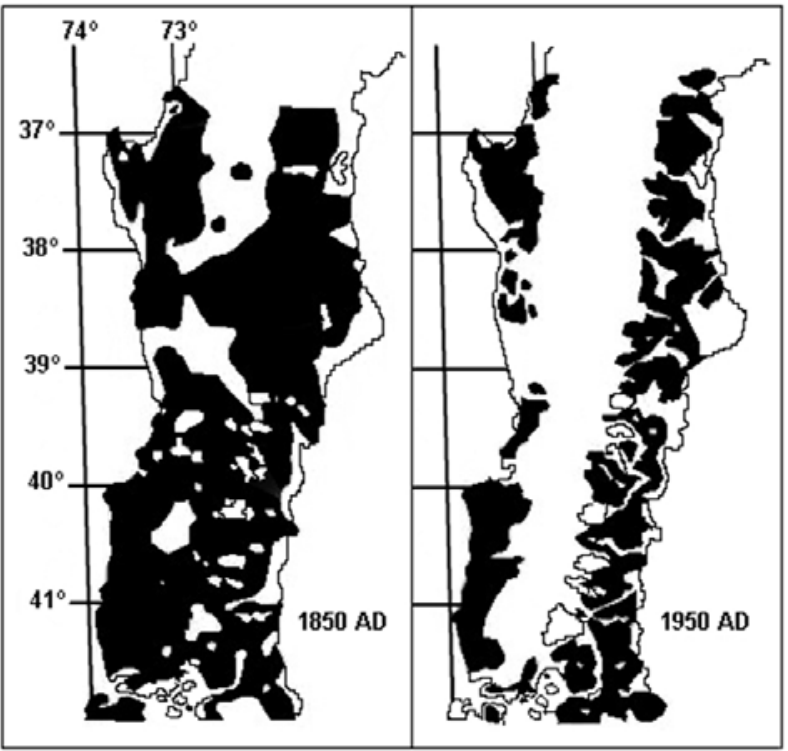

Figura 2. Mapa parcial de Chile. A la izquierda se muestra la cobertura del bosque en 1850. A la derecha se muestra la cobertura de los bosques en 1950 tras la expansión poblacional y los cambios de uso de suelo a raíz de la ganadería y los monocultivos de Eucalyptus y pino. Figura tomada de Heusser (2003).

Figure 2. Partial map of Chile. At left is shown the forest cover at 1850 AD. Forest cover at 1950 is shown at the right after the large expansion of human population and land use changes as a result of animal husbandry and the monoculture of Eucalyptus and pine. Figure taken from Heusser (2003).

importancia, especialmente al considerar que factores de discontinuidad poblacional asociados al calentamiento global aceleran la pérdida de diversidad de lagartos (Sinervo et al. 2010).

\section{AGRADECIMIENTOS}

Paige Alsbury, José Nuñez, Paulo Donoso por su ayuda colectando ejemplares. Herman Núñez realizó comentarios sobre una versión preliminar de este trabajo. NSF-OISE0530267 Speciation in Patagonia: establishing sustainable international collaborations in evolution, ecology, and conservation biology. DIUC-Semilla 2009-016-SP. FONDECYT-1090664. Muestreos autorizados por SAG. Resolución 5347.

\section{BIBLIOGRAFIA}

Ávila, L.J., Frutos, N., Morando, M., Pérez, C.H.F. \& KozyKariski, M. 2006. Iguania, Liolaemini, Liolaemus petrophilus and Liolaemus pictus: distribution extension, filling gaps, new records. Checklist 2(2):65-69.

BELL, K.E. \& DonNELY, M.A. 2006. Influence of forest fragmentation on community structure of frogs and lizards in Northeastern Costa Rica. Conservation Biology 20(6):1750-1760.
Bourke SAzo, J.E. 2005. El efecto de la insularidad sobre la morfología y la variación isoenzimática en dos especies del género Liolaemus (Liolaemidae). Seminario de título. Universidad de Concepción. Concepción, Chile.

Donoso-Barros, R. 1961. The reptiles of the Lund University Chile Expedition. Copeia 4:486-488.

Donoso-Barros, R. 1966. Reptiles de Chile. Ediciones de la Universidad de Chile, Santiago, Chile. 458 pp.

Elgueta, E., Reid, S., Pliscoff, P., M.A., NúÑez, J. \& SmithRamírez, C. 2006. Catastro de vertebrados terrestres y análisis en seis hábitats presentes en la reserva nacional Futaleufú, Provincia de Palena, X Región, Chile. Gayana 70(2):195-205.

Etheridge, R. \& EspinozA, R.E. 2000. Taxonomy of Liolaeminae (Squamata: Iguania: Tropiduridae) and a semi-annotated bibliography. Smithsonian Herpetological Information Service 126:1-64.

Haig, I.T., Teesdale, L.V., Briegler, P.A., Payne, B.H. \&. Haertel, M.H. 1946. Forest resources of Chile, Washington, DC, USA. US Forest Service, Department of Agriculture, in cooperation with Corporación de Fomento de la productividad. $256 \mathrm{pp}$.

Hellmich, W. 1934. Die Eidechsen Chile, Insbesondere die Gattung Liolaemus. Abh Bayer Akad Wiss 24. 140 pp.

Hellmich, W. 1938. Beiträge zur Kenntnis der Herpetofauna Chiles. XII Die Eidechsen des Volcán Villarica. Zoologischer Anzeiger 124:37-249.

Heusser, C.J. 2003. Ice age southern Andes: A chronicle of paleoecological events. Developments in Quaternary 
Science, 3. (Ed. Rose, J). Elsevier. 240 pp.

IBARRA-VIDAL, H. 1989. Impacto de las actividades humanas sobre la herpetofauna en Chile. Comunicaciones del Museo Regional de Concepción 3:33-39.

Labra, A., Pienaar, J. \& Hansen, T.F. 2010. Evolution of thermal physiology in Liolaemus lizards: adaptation, phylogenetic inertia, and niche tracking. The American Naturalist 174(2):204-220.

Ley 19473. Decreto Supremo N ${ }^{\circ}$. Diario oficial de la república, Enero de 1998. Santiago, Chile.

Medel, R.G., Marquet, P.A. \& F.M. JAKSIC. 1988. Microhabitat shifts of lizards under different contexts of sympatry: a case study with South American Liolaemus. Oecología 76(4):567-569.

Müller, L. \& Hellmich, W. 1939. Liolaemus-Arten aus demwestlichen Argentinien (Ergebnisse der vierten Forschungsreise von Prof. Dr. K. Krieg nach Südamerika). IV. Über Liolaemus-Arten aus den Territorien Rio Negro und Neuquen. Zoologischer Anzeiger, Leipzig 128(1/2):1-17.

NúÑEZ, H. 1992. Geographical data of chilean lizards and snakes in the Museo Nacional de Historia Natural, Santiago, Chile. Smithsonian Herpetological Information Service 91:1-9.

NúÑez, H., Maldonado, V. \& Pérez, R. 1997. Reunión de trabajo con especialistas en herpetología para categorización de especies según estado de conservación. Noticiario mensual del Museo Nacional de Historia Natural (Santiago, Chile) 329:12-19.

Ortiz, J.C. 1974. Reptiles del Parque Nacional Vicente Pérez Rosales. I. Hábitos alimenticios de Liolaemus pictus pictus, Dúmeril et Bibron (Squamata - Iguanidae). Anales del Museo Nacional de Historia Natural de Valparaiso 7:317-326.

Ortiz, J.C., Troncoso, J.F., Ibarra-Vidal, H \& NúÑEz, H. 1990. Lista Sistemática, distribución, estados de conservación y clave para los herpetozoos de la VIII Región, Chile. Comunicaciones del Museo Regional de Concepción 4:3143.

Pincheira-Donoso, D. \& NúÑEz, H. 2005. Las especies chilenas del género Liolaemus Wiegmann, 1834 (Iguania Tropiduridae: Liolaeminae). Taxonomía, sistemática y evolución. Publicación ocasional del Museo Nacional de Historia
Natural, Chile 59:7-486,

Sinervo, B., Méndez de la Cruz, F., Miles, D., Heulin, B., Bastiaans, E., Villagrán-Santa Cruz, M., Lara-Resendiz, R., Martínez-Méndez, N., Calderón-Espinoza, M.L., Meza-Lázaro, R.N., Gadsden, H., Avila, L.J., Morando, M., De-la-Riva, I.J., Victoriano-Sepúlveda, P., Duarte Rocha, C.F., Ibargüengoytía, N., Aguilar-Puntriano, C., Massot, M., Lepetz, V., Oksanen, T.A., Chapple, D.G., Bauer, A.M., Branch, W.R., Clobert, J. \& Sites Jr., J. W. 2010. Erosion of lizard diversity by climate change and altered thermical niches. Science 328:894-899.

Smith-Ramírez, C., Armesto, J.J. \& Valdovinos, C. 2005. Historia, biodiversidad y ecología de los bosques costeros de Chile. Editorial Universitaria. Santiago, Chile. 708 pp.

Veloso, A. \& J. NaVArro. 1988. Lista sistemática y distribución geográfica de anfibios y reptiles de Chile. Bolletino del Museo Regionale Scienzia Naturali, Torino 6:481-539.

VidAL, M.A., Veloso,A.\& MÉNDEZ, M. 2006. Insular morphological divergence in the lizard Liolaemus pictus (Liolaemidae). Amphibia-Reptilia 27:103-111.

VidAL, M.A. 2007. Variación geográfica en Liolaemus pictus (Liolaeminae) del archipiélago de Chiloé: el rol de las glaciaciones y la insularidad en la divergencia molecular, fisiológica y morfológica. Tesis de grado. Universidad de Chile. Santiago, Chile.

VidAL, M.A. \& SABAT, P. 2010. Stable isotopes document mainland-island divergence in resource use without concomitant physiological changes in the lizard Liolaemus pictus. Comparative biochemistry and physiology Part B: Biochemistry and Molecular Biology 156:61-67.

Vitousek, P.M.H., Mooney, H.A., Lubchenco, J. \& Mellillo, J.M. 1997. Human domination on Earth's ecosystem. Science 277:494-499.

Willson, M. F., Smith-Ramirez, C., Sabag, C. \& Hernández, J. F. 1996a. Mutualismos entre plantas y animales en bosques templados de Chile. En: Ecología de los bosques nativos de Chile (Eds. Armesto, J.J., Villagrán, C \& C. Kalin Arroyo), pp. 251-254. Ediciones Universidad de Chile, Santiago.

Willson, M. F., Sabag, C., Figueroa, J., Armesto, J.J. \& Caviedes, M. 1996b. Seed dispersal by lizards in Chilean rainforest. Revista Chilena de Historia Natural 69:339-342.

\section{APÉNDICE 1}

Sitios de colecta, considerando tanto puntos con presencia como ausencia de Liolaemus pictus. ${ }^{*}$ muestras tomadas de Museos. $0=$ ausencia de L. pictus, $1=$ presencia histórica de L. pictus y $2=$ presencia actual de L. pictus.

\begin{tabular}{cclcl}
\hline Latitud & Longitud & Localidad & Status & Fuente \\
\hline$-35,05000$ & $-71,28333$ & $7 \mathrm{~km}$ al sur de Curicó (Cbs La laguna) & 0 & Este trabajo \\
$-35,33333$ & $-71,63333$ & $15 \mathrm{~km}$ al Noroeste de Talca & 0 & Este trabajo \\
$-35,33333$ & $-72,40000$ & Constitucion & 0 & Este trabajo \\
$-35,34083$ & $-71,63805$ & NOT1 & 0 & Este trabajo \\
$-35,37234$ & $-71,86633$ & PC17 & 0 & Este trabajo \\
$-35,41673$ & $-71,73143$ & TP16 & 0 & Este trabajo \\
$-35,55000$ & $-71,20000$ & F8 & 0 & Este trabajo \\
$-35,56667$ & $-72,03333$ & Parral & 0 & Este trabajo \\
$-35,58000$ & $-72,02917$ & SJ & 0 & Este trabajo \\
\hline
\end{tabular}




\begin{tabular}{|c|c|c|c|c|}
\hline Latitud & Longitud & Localidad & Status & Fuente \\
\hline$-35,58333$ & $-71,08333$ & Alto Vilches & 0 & Este trabajo \\
\hline$-35,60000$ & $-71,05000$ & Altos de Vilches & 1 & Vidal Maldonado, 2007 \\
\hline$-35,60000$ & $-71,08333$ & Vilches alto & 1 & Núñez, 1992* \\
\hline$-35,60372$ & $-71,73611$ & SJ & 0 & Este trabajo \\
\hline$-35,75000$ & $-71,56667$ & Yerbas Buenas & 0 & Este trabajo \\
\hline$-35,83333$ & $-71,56667$ & Cementerio Linares & 0 & Este trabajo \\
\hline$-35,83333$ & $-72,06667$ & Toribio & 0 & Este trabajo \\
\hline$-35,83528$ & $-72,03463$ & L08 & 0 & Este trabajo \\
\hline$-35,85000$ & $-71,60000$ & Linares & 0 & Este trabajo \\
\hline$-35,85000$ & $-71,25000$ & F9 & 0 & Este trabajo \\
\hline$-35,90000$ & $-71,28333$ & Embalse Ancoa Prec Linares & 0 & Este trabajo \\
\hline$-35,90000$ & $-71,28333$ & F9 & 0 & Este trabajo \\
\hline$-35,91666$ & $-71,36666$ & F9 & 0 & Este trabajo \\
\hline$-35,92336$ & $-72,37012$ & PTC19 & 0 & Este trabajo \\
\hline$-36,13333$ & $-72,76667$ & Cobquecura & 0 & Este trabajo \\
\hline$-36,38793$ & $-72,50395$ & QN21 & 0 & Este trabajo \\
\hline$-36,43333$ & $-72,68333$ & Cementerio de Coelemu & 0 & Este trabajo \\
\hline$-36,55029$ & $-71,54495$ & L10 & 0 & Este trabajo \\
\hline$-36,60000$ & $-72,11667$ & Cementerio de Chillán & 0 & Este trabajo \\
\hline$-36,60000$ & $-71,45000$ & Puquios1 & 0 & Este trabajo \\
\hline$-36,60111$ & $-72,12472$ & $\mathrm{CCH} 2$ & 0 & Este trabajo \\
\hline$-36,61667$ & $-71,43333$ & Puquios & 0 & Este trabajo \\
\hline$-36,61856$ & $-71,43770$ & L12 & 0 & Este trabajo \\
\hline$-36,63333$ & $-73,06667$ & Quiriquina & 0 & Este trabajo \\
\hline$-36,65000$ & $-71,35000$ & Caracol & 0 & Este trabajo \\
\hline 36,73333 & $-73,15000$ & $\mathrm{CN} 22$ & 0 & Este trabajo \\
\hline$-36,76583$ & $-73,14833$ & LE12 & 0 & Este trabajo \\
\hline$-36,78333$ & $-72,91667$ & Nonguen & 0 & Este trabajo \\
\hline$-36,78333$ & $-71,76667$ & Recinto & 1 & Hellmich, 1934* \\
\hline$-36,79138$ & $-73,15694$ & LE12b & 0 & Este trabajo \\
\hline$-36,79583$ & $-73,15598$ & $\mathrm{PZ}$ & 0 & Este trabajo \\
\hline$-36,79806$ & $-73,04361$ & GA & 0 & Este trabajo \\
\hline$-36,81666$ & $-72,66666$ & $\mathrm{FC} 2$ & 0 & Este trabajo \\
\hline$-36,81667$ & $-73,03333$ & Universidad de Concepcion & 0 & Este trabajo \\
\hline$-36,81667$ & $-71,25000$ & Termas de Chillán & 1 & Bourke Suazo, 2005 \\
\hline$-36,83417$ & $-73,03111$ & $\mathrm{UC}$ & 0 & Este trabajo \\
\hline$-36,83667$ & $-73,10361$ & $\mathrm{HF}$ & 0 & Este trabajo \\
\hline$-36,88333$ & $-71,56666$ & E11 & 0 & Este trabajo \\
\hline$-36,88333$ & $-71,56667$ & Las Trancas & 0 & Este trabajo \\
\hline$-36,89444$ & $-71,47472$ & VT1 & 0 & Este trabajo \\
\hline$-36,89450$ & $-71,47472$ & Valle Las Trancas, camino a Shangrilá & 2 & Este trabajo \\
\hline$-36,90000$ & $-71,46667$ & Las Trancas & 0 & Este trabajo \\
\hline$-36,98333$ & $-73,10000$ & Coronel & 0 & Este trabajo \\
\hline$-37,01666$ & $-73,13333$ & B11 & 0 & Este trabajo \\
\hline$-37,07778$ & $-73,11500$ & Curanilahue & 0 & Este trabajo \\
\hline$-37,08277$ & $-72,43611$ & MG3 & 0 & Este trabajo \\
\hline$-37,08333$ & $-73,16667$ & Playa Negra Coronel & 0 & Este trabajo \\
\hline$-37,23333$ & $-71,90000$ & Huepil - Trupan & 0 & Este trabajo \\
\hline$-37,27417$ & $-71,94389$ & TU & 0 & Este trabajo \\
\hline
\end{tabular}




\begin{tabular}{|c|c|c|c|c|}
\hline Latitud & Longitud & Localidad & Status & Fuente \\
\hline$-37,28333$ & $-71,93333$ & Cementerio Tucapel & 0 & Este trabajo \\
\hline$-37,33333$ & $-73,50000$ & Nahuelbuta & 1 & Vidal et al., 2006* \\
\hline$-37,33333$ & $-73,50000$ & Cord. De Nahuelbuta & 1 & Smith-Ramírez et al., 2005 \\
\hline$-37,38540$ & $-71,47481$ & LJ11 & 0 & Este trabajo \\
\hline$-37,45000$ & $-72,35000$ & Los Angeles & 0 & Este trabajo \\
\hline$-37,46667$ & $-72,31667$ & Cementerio Los Angeles & 0 & Este trabajo \\
\hline$-37,56667$ & $-72,03333$ & San Javier & 0 & Este trabajo \\
\hline$-37,78333$ & $-73,05000$ & Parque Nahuelbuta & 2 & Este trabajo \\
\hline$-37,80000$ & $-72,71667$ & Angol (F. Alvarado) & 0 & Este trabajo \\
\hline$-37,81667$ & $-71,66667$ & Hostería Doña Pola (Ralco) & 0 & Este trabajo \\
\hline$-37,81805$ & $-71,67604$ & POLA & 0 & Este trabajo \\
\hline$-37,88333$ & $-72,73333$ & Nahuelbuta & 1 & Bourke Suazo, 2005 \\
\hline$-37,88333$ & $-72,73333$ & Cordillera de Nahuelbuta & 1 & Bourke Suazo, 2005* \\
\hline$-37,88333$ & $-71,18333$ & Butamalal & 1 & Bourke Suazo, 2005* \\
\hline$-37,88333$ & $-71,60000$ & Ralco camino a represa & 0 & Este trabajo \\
\hline$-37,93333$ & $-72,50000$ & R.N. Malleco & 1 & Bourke Suazo, 2005 \\
\hline$-37,93333$ & $-72,50000$ & R.N. malleco & 1 & Bourke Suazo, 2005* \\
\hline$-37,96667$ & $-73,23333$ & Camino a Contulmo (Lago Lanalhue) & 0 & Este trabajo \\
\hline$-38,01667$ & $-73,15000$ & M. N. Contulmo & 0 & Este trabajo \\
\hline$-38,01667$ & $-73,15000$ & $\mathrm{~S}$ de $\mathrm{M} . \mathrm{N}$ Contulmo & 0 & Este trabajo \\
\hline$-38,01878$ & $-73,16500$ & Puren & 2 & Este trabajo \\
\hline$-38,02724$ & $-73,15445$ & M02 & 0 & Este trabajo \\
\hline$-38,02725$ & $-73,15444$ & Puren & 2 & Este trabajo \\
\hline$-38,05000$ & $-71,71667$ & Termas de Pemehue & 0 & Este trabajo \\
\hline$-38,10000$ & $-73,26667$ & Lago Lanalhue & 0 & Este trabajo \\
\hline$-38,11667$ & $-73,33333$ & Lago Lleu Lleu (Camping Rayen Antu) & 0 & Este trabajo \\
\hline$-38,12583$ & $-73,34500$ & LLU14 & 0 & Este trabajo \\
\hline$-38,13333$ & $-73,35000$ & Lago Lleu Lleu Camping Rayenco & 0 & Este trabajo \\
\hline$-38,16667$ & $-71,80000$ & $2 \mathrm{Km}$ fuera RN Malleco & 0 & Este trabajo \\
\hline$-38,16667$ & $-71,95000$ & Niblinto cruce Victoria & 0 & Este trabajo \\
\hline$-38,20000$ & $-72,15000$ & 20 km W P. N. Tolhuaca & 0 & Este trabajo \\
\hline$-38,20000$ & $-71,83333$ & RN Malleco Las Mentas & 0 & Este trabajo \\
\hline$-38,20000$ & $-71,83333$ & R.N.Malleco Las Mentas & 2 & Este trabajo \\
\hline$-38,21667$ & $-71,80000$ & Entrada P. N Tolhuaca & 0 & Este trabajo \\
\hline$-38,21667$ & $-71,80000$ & PN Tolhuaca & 0 & Este trabajo \\
\hline$-38,21667$ & $-71,86667$ & Lag. Malleco & 1 & Medel et al., 1988 \\
\hline$-38,21803$ & $-71,80950$ & Lonquimay & 2 & Este trabajo \\
\hline$-38,21803$ & $-71,81236$ & Lonquimay & 2 & Este trabajo \\
\hline$-38,36667$ & $-71,71667$ & Puesco & 1 & Bourke Suazo, 2005* \\
\hline$-38,38000$ & $-73,91667$ & R.N. Isla Mocha & 1 & Bourke Suazo, 2005* \\
\hline$-38,40852$ & $-72,78040$ & $\mathrm{GV}$ & 0 & Este trabajo \\
\hline$-38,41667$ & $-72,83333$ & R.N. Huerquehue & 1 & Bourke Suazo, 2005* \\
\hline$-38,42027$ & $-71,54416$ & RM10 & 0 & Este trabajo \\
\hline$-38,46181$ & $-71,74208$ & Salto El indio & 2 & Este trabajo \\
\hline$-38,46822$ & $-71,51081$ & R. N. Malalcahuello & 2 & Este trabajo \\
\hline$-38,50000$ & $-71,18333$ & R.N. Malalcahuello & 1 & Bourke Suazo, 2005* \\
\hline$-38,55000$ & $-71,45000$ & Camino a Lonquimay & 0 & Este trabajo \\
\hline$-38,55924$ & $-71,45142$ & CLQ05 & 0 & Este trabajo \\
\hline$-38,66667$ & $-71,88333$ & Ref. Volcan Llaima & 0 & Este trabajo \\
\hline
\end{tabular}


Distribución de Liolaemus pictus: IVÁN M. Vera-EsCALONA ET AL.

\begin{tabular}{|c|c|c|c|c|}
\hline Latitud & Longitud & Localidad & Status & Fuente \\
\hline$-38,68333$ & $-71,80000$ & Cherquenco Volcan Llaima & 0 & Este trabajo \\
\hline$-38,68611$ & $-71,80056$ & Ref.Vn.Llaima & 2 & Este trabajo \\
\hline$-38,71667$ & $-72,56667$ & Nahuelbuta & 0 & Este trabajo \\
\hline$-38,71667$ & $-71,71667$ & Volcán Llaima & 1 & Bourke Suazo, 2005 \\
\hline$-38,71667$ & $-71,71667$ & Volcán Llaima & 1 & Bourke Suazo, 2005* \\
\hline$-38,73333$ & $-72,58333$ & Villa Cautín (Puente Cautin) & 0 & Este trabajo \\
\hline$-38,73333$ & $-72,60000$ & Temuco & 1 & Hellmich, 1934* \\
\hline$-38,78333$ & $-73,38333$ & Cementerio Puerto Saavedra & 0 & Este trabajo \\
\hline$-38,99040$ & $-73,20535$ & M10 & 0 & Este trabajo \\
\hline$-39,18333$ & $-71,75000$ & Cam. Parque Huerquehue & 0 & Este trabajo \\
\hline$-39,20000$ & $-71,76667$ & P.N Huerquehue & 2 & Este trabajo \\
\hline$-39,23333$ & $-71,65000$ & $4 \mathrm{Km}$. O Puesco & 0 & Este trabajo \\
\hline$-39,25000$ & $-72,21667$ & Neltume & 1 & Núñez, 1992* \\
\hline$-39,26667$ & $-71,95000$ & Pucón & 1 & Hellmich, 1934* \\
\hline$-39,36667$ & $-71,70000$ & Cca. de Pucon & 0 & Este trabajo \\
\hline$-39,41667$ & $-72,88333$ & Camino La Capilla & 0 & Este trabajo \\
\hline$-39,41667$ & $-71,76667$ & Cca. salida P. N. Villarrica & 0 & Este trabajo \\
\hline$-39,41667$ & $-71,93333$ & Volcán Villarrica & 1 & Hellmich, 1938* \\
\hline$-39,41667$ & $-71,93333$ & Volcán Villarrica & 1 & Hellmich, 1934* \\
\hline$-39,41667$ & $-71,66667$ & Puesco & 1 & Vidal et al., 2006* \\
\hline$-39,41972$ & $-71,78075$ & Villarrica & 2 & Este trabajo \\
\hline$-39,43333$ & $-71,63333$ & Desv Pucon - Curarrehue & 0 & Este trabajo \\
\hline$-39,46667$ & $-72,85000$ & Cam. Calquinco La Capilla & 0 & Este trabajo \\
\hline$-39,49569$ & $-71,84406$ & Villarrica & 2 & Este trabajo \\
\hline$-39,53333$ & $-71,56667$ & Lago Caburga & 0 & Este trabajo \\
\hline$-39,56667$ & $-72,33333$ & Neltume & 0 & Este trabajo \\
\hline$-39,70000$ & $-71,96667$ & Neltume & 1 & Hellmich, 1934* \\
\hline$-39,74786$ & $-73,34847$ & Camino Parque Oncol & 2 & Este trabajo \\
\hline$-39,78333$ & $-73,26272$ & Isla Teja & 1 & Bourke Suazo, 2005* \\
\hline$-39,81667$ & $-73,23333$ & Valdivia & 1 & Hellmich, 1934* \\
\hline$-39,81667$ & $-73,23333$ & Valdivia & 1 & Núñez, 1992* \\
\hline$-39,88333$ & $-73,41667$ & Corral (Fuerte) & 0 & Este trabajo \\
\hline$-39,98333$ & $-72,81667$ & Reumén & 1 & Hellmich, 1934* \\
\hline$-40,06892$ & $-73,24811$ & Tres chiflones altos & 2 & Este trabajo \\
\hline$-40,14028$ & $-71,47417$ & Camino a Hua Hum & 2 & Este trabajo \\
\hline$-40,16917$ & $-73,46306$ & Cam. Hueicolla. MN Alerce costero & 2 & Este trabajo \\
\hline$-40,18333$ & $-72,00000$ & Pasado los Llolles & 0 & Este trabajo \\
\hline$-40,18611$ & $-72,00500$ & Pasado Los Llolles & 2 & Este trabajo \\
\hline$-40,20000$ & $-72,26667$ & Lago Ranco cca Lifen & 0 & Este trabajo \\
\hline$-40,20222$ & $-72,26833$ & Lago Ranco cca. Llifen & 2 & Este trabajo \\
\hline$-40,21000$ & $-72,10833$ & Camino Lago Maihue & 2 & Este trabajo \\
\hline$-40,26667$ & $-73,31667$ & Camino La Union- Hueicolla & 0 & Este trabajo \\
\hline$-40,26833$ & $-73,32861$ & Camino La Unión a Hueicolla & 2 & Este trabajo \\
\hline$-40,43333$ & $-73,08333$ & Osorno & 1 & Hellmich, 1934* \\
\hline$-40,57503$ & $-73,71100$ & Maicolpué & 2 & Este trabajo \\
\hline$-40,67556$ & $-71,72861$ & Villa Angostura & 2 & Este trabajo \\
\hline$-40,68333$ & $-72,00000$ & H. Puyehue-Ag.Caltes & 2 & Este trabajo \\
\hline$-40,75000$ & $-72,15000$ & Antillanca & 1 & Bourke Suazo, 2005* \\
\hline$-40,76667$ & $-72,26667$ & Antillanca & 1 & Vidal Maldonado, 2007 \\
\hline
\end{tabular}




\begin{tabular}{|c|c|c|c|c|}
\hline Latitud & Longitud & Localidad & Status & Fuente \\
\hline$-40,76667$ & $-72,20000$ & Antillanca & 1 & Vidal et al., 2006* \\
\hline$-40,76944$ & $-72,20250$ & Antillanca & 2 & Este trabajo \\
\hline$-40,78319$ & $-72,21669$ & Puyehue & 2 & Este trabajo \\
\hline$-40,81667$ & $-72,46667$ & L. Rupanco & 1 & Bourke Suazo, 2005* \\
\hline$-40,96667$ & $-72,33333$ & Las cascadas & 1 & Vidal Maldonado, 2007 \\
\hline$-41,05000$ & $-72,61667$ & Las cascadas & 1 & Bourke Suazo, 2005 \\
\hline$-41,05000$ & $-72,61667$ & Las cascadas & 1 & Bourke Suazo, 2005* \\
\hline$-41,11667$ & $-73,38333$ & Petrohué & 1 & Donoso-Barros, 1961* \\
\hline$-41,11670$ & $-71,21670$ & Estancia el condor, Nahuel huapi & 1 & Müller \& Hellmich, 1939 \\
\hline$-41,13667$ & $-71,33861$ & Bariloche & 2 & Este trabajo \\
\hline$-41,26667$ & $-72,23333$ & Cayetue & 1 & Hellmich, 1934* \\
\hline$-41,31667$ & $-72,95000$ & Puerto Varas & 1 & Hellmich, 1934* \\
\hline$-41,33333$ & $-73,41667$ & Los muermos & 1 & Vidal Maldonado, 2007 \\
\hline$-41,44333$ & $-73,68750$ & Estaquillas & 2 & Este trabajo \\
\hline$-41,44833$ & $-73,72694$ & Estaquillas & 2 & Este trabajo \\
\hline$-41,60422$ & $-72,64756$ & Parque Alerce Andino & 2 & Este trabajo \\
\hline$-41,60442$ & $-72,62953$ & Parque Alerce Andino & 2 & Este trabajo \\
\hline$-41,65000$ & $-72,26667$ & Cuenca Río Puelo & 2 & Este trabajo \\
\hline$-41,66667$ & $-72,21667$ & Lago todos los santos & 1 & Hellmich, 1934* \\
\hline$-41,91667$ & $-73,84222$ & Sur de Ancud & 2 & Este trabajo \\
\hline$-41,91667$ & $-72,00000$ & P.N. Hornopirén & 1 & Bourke Suazo, 2005* \\
\hline$-41,91917$ & $-73,91361$ & Sur de Ancud & 2 & Este trabajo \\
\hline$-41,93333$ & $-72,38333$ & Hornopirén & 1 & Vidal Maldonado, 2007 \\
\hline$-41,93333$ & $-73,88333$ & Isla de Chiloé & 1 & Vidal et al., 2006* \\
\hline$-41,93694$ & $-73,89361$ & Sur de Ancud & 2 & Este trabajo \\
\hline$-41,96667$ & $-73,55000$ & Isla de Chiloé & 1 & Bourke Suazo, 2005 \\
\hline$-41,96667$ & $-72,00000$ & Hornopirén & 1 & Bourke Suazo, 2005 \\
\hline$-42,06694$ & $-73,75250$ & Belben & 2 & Este trabajo \\
\hline$-42,11583$ & $-73,81953$ & Alto de Tantauco & 2 & Este trabajo \\
\hline$-42,11964$ & $-73,81100$ & Alto de Tantauco & 2 & Este trabajo \\
\hline$-42,13333$ & $-73,38333$ & Yerbaloza & 1 & Vidal Maldonado, 2007 \\
\hline$-42,13333$ & $-73,58333$ & Quellón & 1 & Vidal Maldonado, 2007 \\
\hline$-42,30000$ & $-73,13333$ & Isla Butachauques & 1 & Vidal Maldonado, 2007 \\
\hline$-42,38333$ & $-73,85000$ & Ruta a Abtao & 1 & Vidal Maldonado, 2007 \\
\hline$-42,38489$ & $-73,91308$ & Pastahué & 2 & Este trabajo \\
\hline$-42,62525$ & $-74,10803$ & Lago Cucao & 2 & Este trabajo \\
\hline$-42,69075$ & $-73,92886$ & Lago Huillinco & 2 & Este trabajo \\
\hline$-42,73333$ & $-72,95000$ & Isla Talcán & 1 & Vidal Maldonado, 2007 \\
\hline$-42,86667$ & $-73,80000$ & Quemchi & 1 & Vidal Maldonado, 2007 \\
\hline$-42,95000$ & $-72,63333$ & Chaitén & 1 & Vidal Maldonado, 2007 \\
\hline$-42,98756$ & $-72,41369$ & Chaitén & 2 & Este trabajo \\
\hline$-42,99178$ & $-72,43439$ & Chaitén & 2 & Este trabajo \\
\hline$-43,04939$ & $-73,87736$ & Parque Tantauco & 2 & Este trabajo \\
\hline$-43,50000$ & $-72,33333$ & Futaleufú & 1 & Elgueta et al. 2006 \\
\hline$-43,58333$ & $-74,68333$ & Isla Guafo & 1 & Vidal Maldonado, 2007 \\
\hline$-43,70225$ & $-71,41044$ & Ruta Corcovado & 1 & Avila et al., 2006 \\
\hline$-43,88356$ & $-72,92925$ & Canal Garrado & 2 & Este trabajo \\
\hline
\end{tabular}

Recibido: 02.06.10

Aceptado: 03.11.10 